\title{
Un contexto geográfico para la investigación demográfica de la frontera norte*
}

\author{
René M. Zenteno Quintero y Rodolfo Cruz Piñeiro**
}

\begin{abstract}
Antes de iniciar un estudio demográfico de la frontera norte es imprescindible delimitar el ámbito geográfico que será el objeto de medición y análisis. En este artículo se aborda ese tema, proponiendo que se defina el contexto geográfico fronterizo tomando como unidad de análisis los municipios colindantes con Estados Unidos. Para sustentar la propuesta se exponen las ventajas comparativas de privilegiar a los municipios sobre las entidades federativas como unidad de análisis; se señala la heterogeneidad de los niveles de desarrollo que presenta la franja fronteriza; y se profundiza en el análisis de los fenómenos demográficos.
\end{abstract}

Al iniciar un programa de estudios demográficos para la frontera norte de México resulta de suma importancia, entre otras cosas, definir el ámbito geográfico a través del cual se van a medir y estudiar sus características sociodemográficas. ${ }^{1}$ Este esfuerzo metodológico que pudiera parecer sencillo enfrenta dos problemas fundamentales. El primero de ellos se refiere a la multiplicidad de criterios con que se ha delimitado el espacio de los fenómenos fronterizos. El segundo, relacionado estrechamente con el anterior, tiene que ver con el presunto carácter de región que se le ha atribuido a esta parte del país.

Si observamos las divisiones regionales más importantes que se han realizado para el estudio de las desigualdades en México, es notoria la inexistencia de alguna zona denominada "fronteriza del norte de México" (Bataillon, 1969; Unikel et al., 1976; Bassols, 1978, y Coplamar, 1982). En términos generales estos trabajos han tomado como unidad de análisis a las entidades federativas y han fragmentado el amplio norte de México en dos o tres regiones geoeconómicas.

Por otro lado, en los estudios que hacen referencia directa a una región fronteriza del norte de México existe - en general-- una gran heterogeneidad con respecto a la delimitación de esta zona. El universo de

* Este trabajo, con algunas modificaciones, fue presentado en el Simposio Binacional sobre Población en la Región Fronteriza México-Estados Unidos que se llevó a cabo en la ciudad de Tijuana en junio de 1987. A la vez quisiéramos agradecer los comentarios al trabajo hechos por Tito Alegría, Beatriz Figueroa y Brígida García, así como el apoyo de cómputo brindado por Nayin Muñiz.

* Investigadores de El Colegio de la Frontera Norte.

1 Ante la carencia de un cuerpo estructurado de investigaciones demográficas cuyo objeto de estudio fuera la frontera norte de México, y dada la relevancia de este tipo de estudios para la región, El Colegio de la Frontera Norte dio inicio en 1987 a su actual Departamento de Estudios de Población. 
estudio definido varía enormemente. En algunos de los trabajos se ha tomado como base a las entidades federativas del norte de México; en otros, a los municipios que colindan físicamente con Estados Unidos, y en algunos más las principales localidades urbanas fronterizas. ${ }^{2}$

A unque reconocemos que el ámbito geográfico en que toman lugar las interacciones sociales, culturales, económicas, demográficas, etc., fronterizas, puede ser definido de acuerdo a los objetivos de cada investigación, hacer alusión directa a una región conlleva el reconocimiento de elementos estructurales comunes, así como la utilización de criterios de regionalización que permitan definir a esta parte del país como una zona socioeconómicamente homogénea y físicamente integrada. Al respecto, la región fronteriza ha sido identificada más por la existencia de situaciones singulares que por criterios de regionalización.

Una de estas particularidades radica en su colindancia con Estados Unidos. La conformación histórica de la frontera entre dos países ampliamente diferenciados, y las contradicciones propias del desarrollo mexicano, han dado lugar a un espacio cuya estructura socioeconómica destacan algunos autores por su escasa integración a la economía nacional, y en contrapartida por su alto grado de interdependencia o dependencia con Estados Unidos (Urquidi y Méndez, 1975; García, 1981; Victoria, 1982; Ojeda, 1982; Fernández, 1983):

Frontera reveladora del subdesarrollo, ella acentúa las características propias de toda economía dependiente, cuya supervivencia es posible únicamente por su integración a la moderna división internacional del trabajo (Revel-Mouroz, 1984:11).

Otro de los elementos que han propiciado el reconocimiento de una problemática diferente en la frontera norte ha sido la existencia de condiciones especiales para su desarrollo. Desde fines del siglo pasado los distintos gobiernos federales han promulgado reglamentaciones (como los regímenes de zonas y perímetros libres], y programas económicos y sociales particulares para esta zona. ${ }^{3}$

No obstante que estas circunstancias posibilitan el reconocimiento de una situación regional (nacional o binacional) en la frontera norte de México, pocos son los esfuerzos teóricos que se han realizado para escudriñar en sus elementos estructurales. La mayoría de los trabajos han preferido definir a esta región a partir de las problemáticas especificas que surgen

\footnotetext{
${ }^{2}$ Un resumen de los principales trabajos que han tomado como base las entidades federativas o los municipios fronterizos puede verse en Fernández y Tamayo (1983) y Margulis y Tuirán (1986).

${ }^{3}$ Un buen análisis y explicación de la formación de zonas y perímetros libres en la frontera norte de México, así como de los principales programas para su desarrollo pueden verse en los trabajos de Fernández y Tamayo (1983) y Mendoza Berrueto (1982).
} 
de dicha estructura como son el alto crecimiento demográfico, el fuerte desarrollo de la industria maquiladora, el alto intercambio comercial de bienes y servicios con Estados Unidos, etcétera.

A pesar de la existencia de características singulares en esta parte del país, el problema principal para considerarla como una sola región geoeconómica estriba en la nula integración que existe entre las zonas que la conforman. Las causas que han propiciado esta situación son, en primer término, las condiciones físico-geográficas del norte de México, que dificultan las relaciones este-oeste entre las distintas áreas y ciudades fronterizas; y en segundo término, que las interacciones económicas y sociales predominantes de estas zonas se hayan establecido históricamente en dirección norte-sur con regiones del suroeste norteamericano y, secundariamente, con el centro de México.

Un aspecto más que complejiza la identificación de la frontera norte como una región uniforme es su heterogeneidad interna en términos de desarrollo socioeconómico. Como se ha señalado, el único elemento común indiscutible de homogeneidad fronteriza es su desigualdad manifiesta con respecto a los niveles de desarrollo estadunidense (Fernández y Tamayo, 1983).

Por las razones antes expuestas (escasa integración y heterogeneidad interna) cualquier delimitación que pretenda considerar esta parte del país como una región presenta problemas; consideramos que ésta más bien debe ser tomada como un contexto geográfico para el estudio de los fenómenos fronterizos. Preferimos denominarla de dicha manera, ya que el criterio fundamental para definir a esta zona es su colindancia con Estados Unidos.

Una investigación amplia que conjunte los principales hallazgos teóricos en torno a los elementos estructurales que dan pie a una problemática fronteriza (así como criterios de regionalización), deberá poner en claro en un futuro próximo la discusión sobre el cáracter omnímodo de la región fronteriza, o bien, la existencia de una serie de regiones.

En las siguientes páginas trataremos de exponer la importancia que tiene para la investigación demográfica tomar como contexto geográfico fronterizo al conjunto de municipios que colindan con Estados Unidos. Posteriormente intentaremos señalar algunos problemas que pueden presentarse al estudiar esta zona del país como una unidad sin tomar en cuenta para ello sus diferencias socioeconómicas. Como muestra de estas diferencias presentamos un análisis de los niveles de marginación y de algunas características demográficas de los municipios fronterizos.

\section{El contexto geográfico fronterizo: municipios versus estados}

La zona fronteriza definida a partir de la contigüidad física se puede referir al conjunto de las seis entidades federativas del norte de México (Baja 
California, Coahuila, Chihuahua, Nuevo León, Sonora y Tamaulipas), o también a los municipios que se encuentran junto a la línea divisoria con Estados Unidos. No obstante, tomar como unidad de análisis uno u otro para el desarrollo de una investigación demográfica de la frontera norte, puede conducir a obtener conclusiones distintas sobre la evolución y características de sus fenómenos sociodemográficos. Por lo tanto, resulta prioritario acotar cuál es el contexto más adecuado para el estudio de estos fenómenos.

La falta de información desagregada para otras unidades políticoadministrativas, diferentes a las mencionadas, ha limitado en gran medida la utilización de éstas como unidades de estudio para la investigación. Los estudios demográficos no quedan exentos de esta dificultad, ya que la mayoría de los datos sociodemográficos son recopilados y/o publicados únicamente a nivel estatal y municipal. Para las encuestas cabe señalar que muy pocas veces responden a las necesidades específicas de la frontera norte (además que en pocas ocasiones son levantadas en esta parte del país), debido a que éstas se realizan sin tomar en cuenta las particularidades de la zona, puesto que se ha dado mayor importancia a la comparabilidad a nivel nacional que a las diferencias interregionales.

En el presente trabajo privilegiamos como contexto geográfico para el estudio demográfico de la frontera norte de México, al conjunto de treinta y cinco municipios que colindan con Estados Unidos, más los municipios de Ensenada, B.C., Manuel Benavides, Chih. y Valle Hermoso, Tamps., que aunque no cumplen con esta condición son considerados como fronterizos por su gran interacción con Estados Unidos; además, son incluidos en el Programa de Desarrollo de la Frontera Norte (1985). Esto da un total de treinta y ocho municipios, los cuales - tal y como veremos más adelante - resultan unidades de análisis más precisas que los estados del norte de México. Creemos que éste es un primer paso para identificar al interior de este marco zonas con características comunes: demográficas, sociales, económicas, geográficas, etcétera.

En el cuadro 1 se expone la población total de estos municipios de 1930 a 1980. Durante este periodo la población del conjunto de municipios se multiplicó once veces, caracterizándose así como una zona de gran dinamismo demográfico. Entre 1930 y 1970 los municipios fronterizos (de aquí en adelante denominados franja fronteriza (FF)) presentaron tasas de crecimiento superiores a los estados del norte de México (EF) y al total del país (véase cuadro 2 y gráfica 1 ). Al mismo tiempo se visualiza claramente una tendencia a la baja en su tasa de crecimiento. Tan es así que en la década 1970-1980 por primera vez ésta es inferior a la de los estados y a la del país.

Durante los veinte años posteriores a 1940 los municipios fronterizos experimentaron sus tasas de crecimiento más altas en los años analizados. Llama la atención cómo la tasa de éstos, en la década 1940-1950, es 
casi tres veces superior a la experimentada por el país y casi el doble que la de los estados fronterizos. Inclusive podemos decir que el crecimiento de estos últimos se explica en gran medida por los cambios ocurridos en la FF.

Las posibles explicaciones a este significativo crecimiento de la FF deben asociarse a una serie de acontecimientos, tanto internos como externos, que determinaron que la zona ejerciera una fuerte atracción para la emigración durante esta época. ${ }^{4}$ Cabe señalar, por un lado, el auge económico que tuvieron los valles agrícolas de los municipios de Mexicali, Reynosa y Matamoros. Y por el otro, los efectos derivados por el establecimiento del Programa de Braceros (1942-1964) entre los gobiernos de Estados Unidos y México, y la influencia que tuvo la reactivación económica norteamericana, una vez finalizada la segunda guerra mundial, sobre la propia economía de las ciudades fronterizas.

La influencia del Programa de Braceros sobre el crecimiento de las ciudades fronterizas fue en esta época indirecta, ya que el hecho de que se empezaran a reclutar trabajadores en el centro de México para desempenar trabajos mejor remunerados en Estados Unidos, creó la impresión de que el trabajo abundaba, aumentando con esto la migración hacia la frontera y la emigración hacia los Estados Unidos (Driscoll, 1985).

En la década siguiente (1960-1970) la tasa de crecimiento anual de la frontera siguió siendo más alta que la nacional y la de los $\mathrm{EF}$, sin embargo, respecto a los diez años anteriores hubo una disminución significativa de -1.7. Muy probablemente el término del Programa de Braceros en 1964 provocó que esta zona perdiera atracción durante el segundo quinquenio de la década, ya que empezó a hacerse más difícil la venta de fuerza de trabajo en Estados Unidos. Pero por otro lado, este acontecimiento pudo traer como consecuencia también el efecto contrario, es decir, un incremento en la población, si consideramos la hipótesis de que una parte importante de los trabajadores que fueron repatriados por el gobierno de Estados Unidos se quedaron a vivir en la zona fronteriza con la expectativa de volver a laborar en el país vecino. Además, el auge que las zonas agrícolas fronterizas habían manifestado años atrás se vio frenado abruptamente por la caída de los precios internacionales del algodón, cuando este producto era la base principal de su desarrollo. ${ }^{5}$

${ }^{4}$ Si suponemos que la tas ${ }^{-}$de crecimiento natural de la franja fronteriza era igual a la total del pais (la magnitud de esta última está determinada casi en su totalidad por la diferencia entre los nacimientos y defunciones), podemos inferir que aproximadamente $70 \%$ del crecimiento total de la FF se explica por la relación positiva entre la inmigración y la emigración (saldo neto migratorio).

${ }^{5}$ El cultivo del algodón requiere de un uso intensivo de mano de obra durante la época de cosecha. Para cubrir esta labor, las migraciones (especialmente de tipo temporal) desempeñaban un rol importante. 


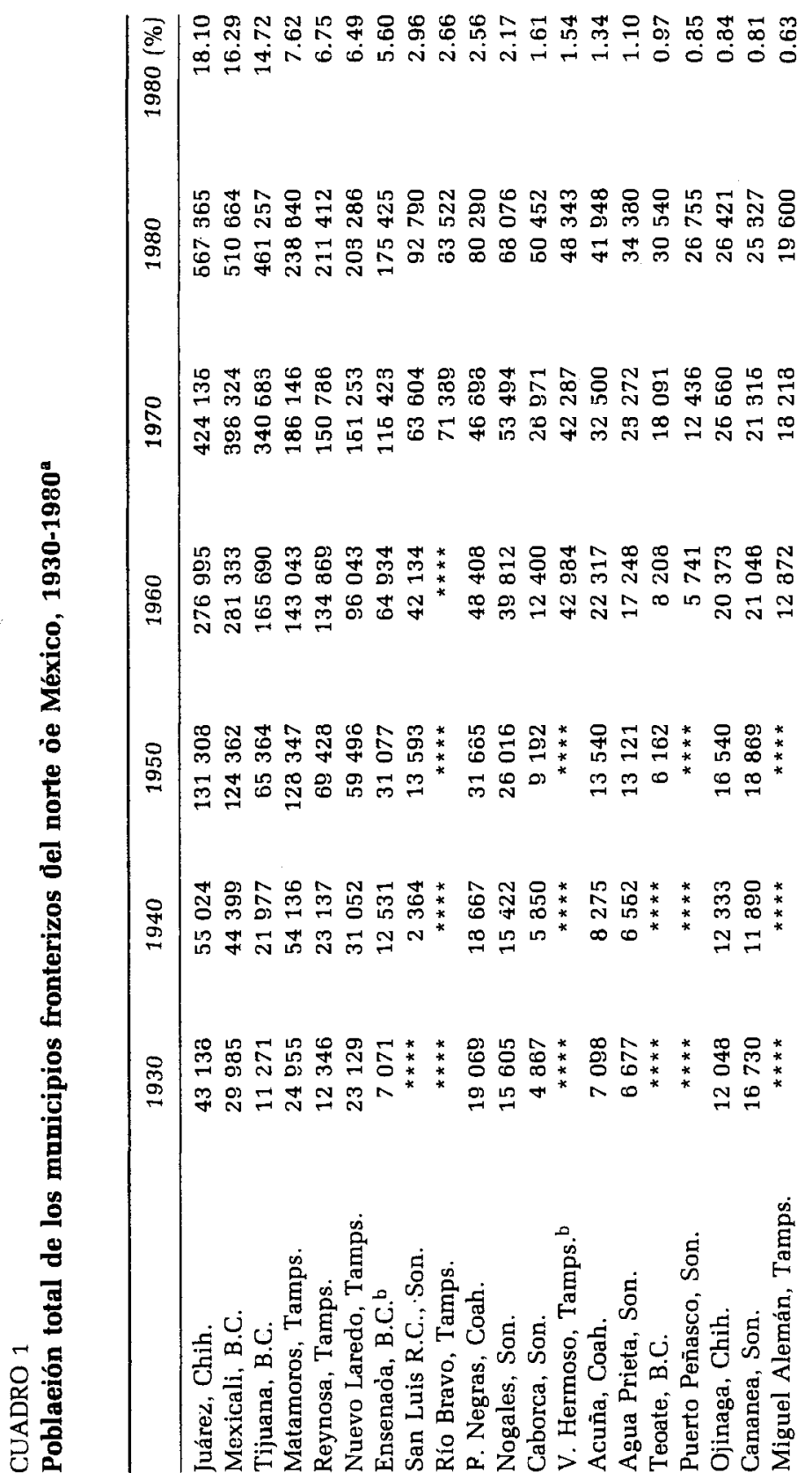




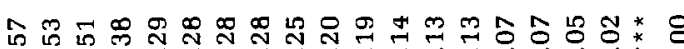

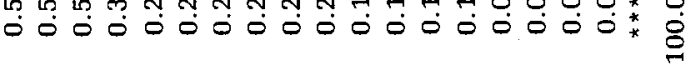

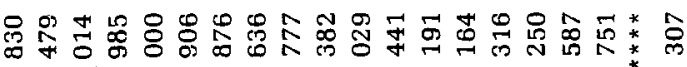

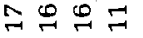

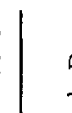

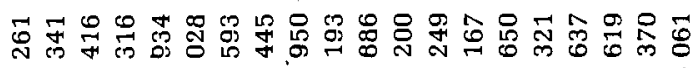

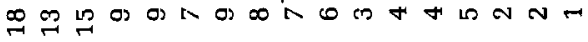

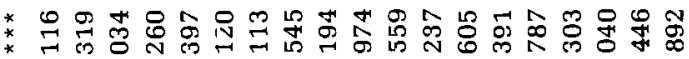

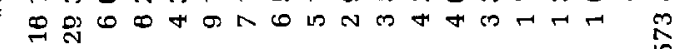

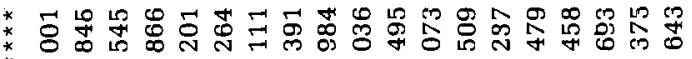

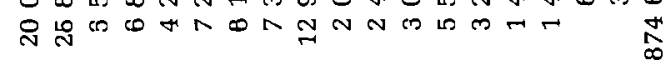

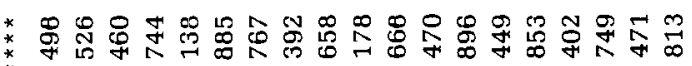

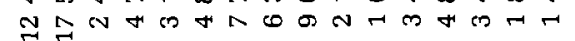

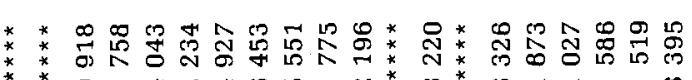

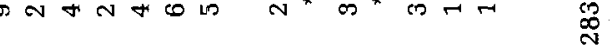

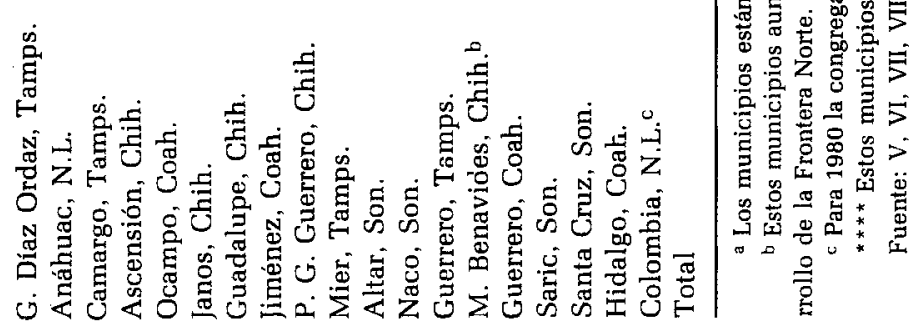


ESTUDIOS DEMOGRÁFICOS Y URBANOS

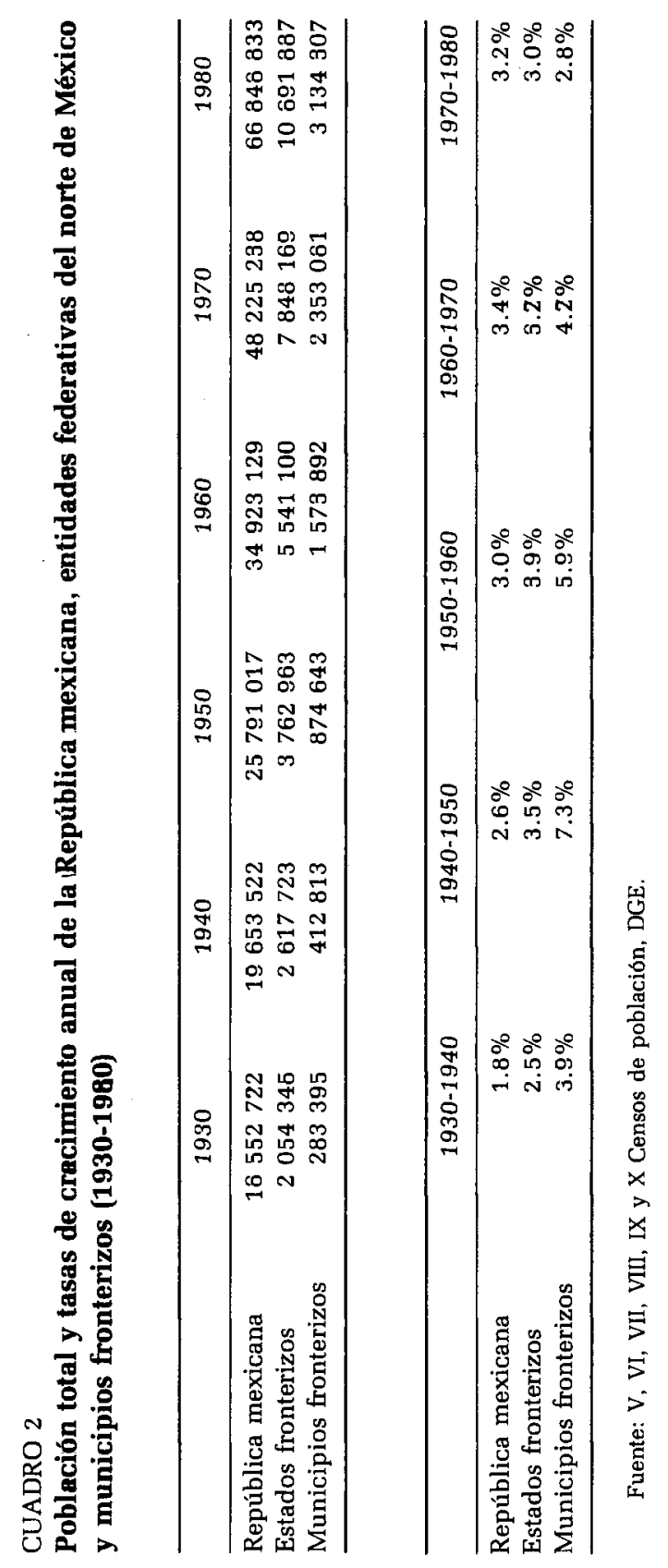


INVESTIGACIÓN DEMOGRÁFICA EN LA FRONTERA NORTE

407

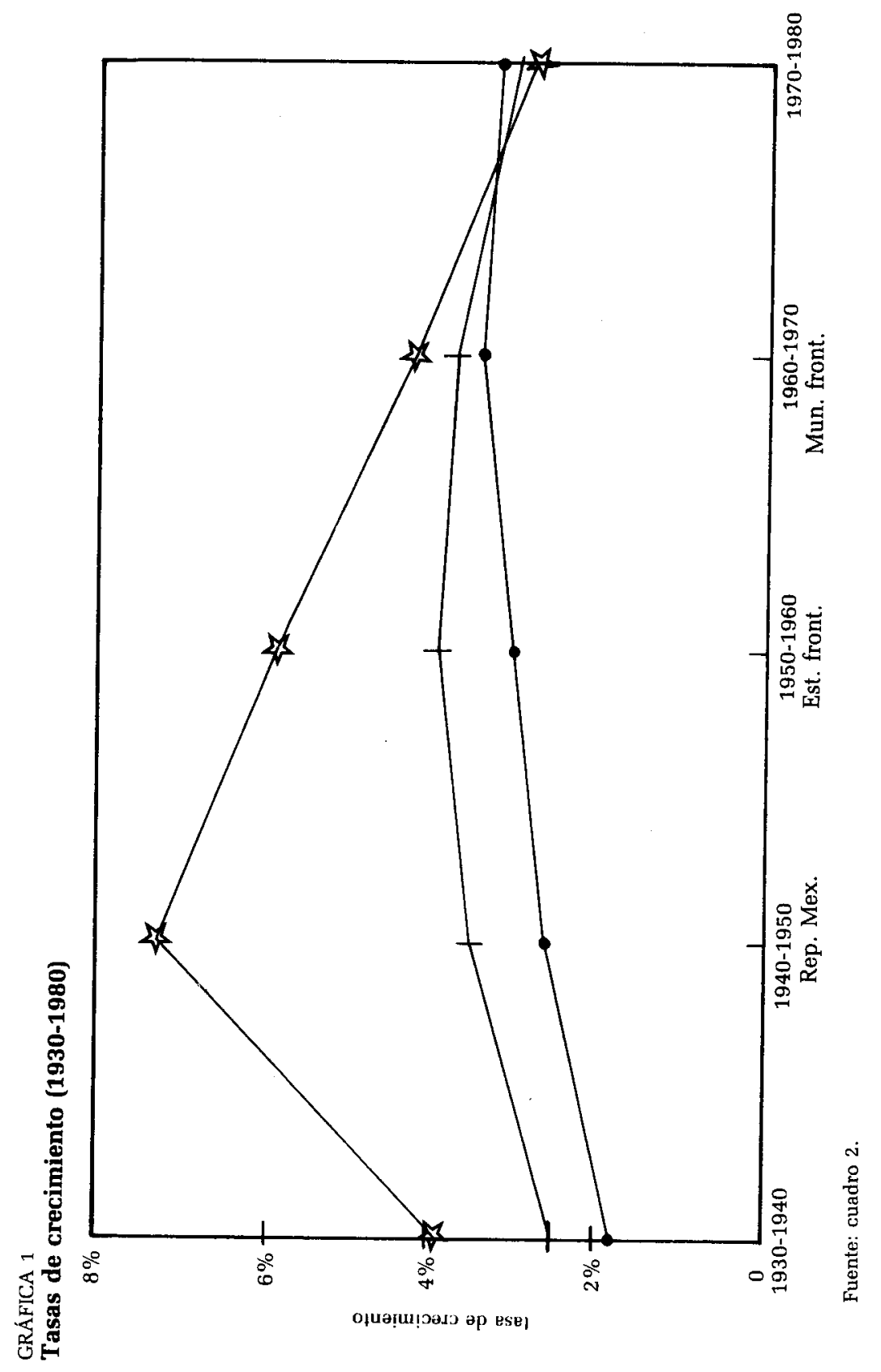


A pesar del incipiente inicio en la disminución del crecimiento demográfico de la FF, se creyó que durante los años setenta su población, y especialmente la de Baja California, seguiría creciendo a tasas elevadas. Empero, los datos arrojados por el Censo de Población y Vivienda de 1980 vinieron a contradecir estas proyecciones, ya que mostraron una caída abrupta en sus niveles de crecimiento.

Para el periodo 1970-1980 el crecimiento tan acelerado que se venía observando en esta zona disminuyó de manera significativa y la tasa alcanzada fue de tan sólo $2.8 \%$. Una de las explicaciones que se han dado a esta disminución es la posible subenumeración de población en el censo de 1980, producida por el concepto de "residente habitual" que se utilizó en dicho censo. Esta hipótesis ha sido ampliamente comentada para el estado de Baja California (Corona, 1986). ${ }^{6}$

Cabe hacer notar que buena parte de esta disminución era de esperarse por las siguientes razones:

i) Los saldos netos migratorios de los municipios fronterizos muy probablemente encontrarían un límite después de la gran tendencia creciente que habían manifestado históricamente, sobre todo a raíz de que las posibilidades de encontrar empleo en Estados Unidos se habían agotado casi por completo como consecuencia de la finalización del Programa de Braceros.

ii) Los efectos que tuvo la finalización del Programa de Braceros sobre el descenso de la inmigración a la frontera (y por ende de su crecimiento) difícilmente se reflejó en toda su magnitud con la tendecia mostrada entre 1960 y 1970, ya que este hecho tomó lugar a mediados de la década, es decir, durante la primera mitad del periodo las condiciones externas que hacían atractiva a esta zona aún existían. Por lo tanto, la tasa de crecimiento 1970-1980 evidenció más claramente los efectos derivados de la terminación del programa.

iii) La contribución relativa de las migraciones a una población mucho más extensa que en décadas anteriores era de esperar que descendiera, aún cuando éstas pudieran haber aumentado en números absolutos.

iv) Por último, no pueden soslayarse los efectos que la política nacional demográfica ha tenido, junto con una actitud creciente de las parejas por controlar su fecundidad, sobre la disminución en las tasas de natalidad - y crecimiento- del país. Esta realidad que tuvo lugar más clara-

6 Vale la pena señalar que los resultados de la Encuesta Demográfica de Baja California (1986) confirmaron la subestimación del censo de 1980 en la entidad, particularmente en el caso del municipio de Tijuana. A pesar de esto no existen aún elementos suficientes para explicar claramente cuáles fueron las causas que produjeron este problema. 
mente a mediados de la década de los setenta a nivel nacional, ya se había venido detectando con anterioridad en el norte del país. ${ }^{7}$

Como se mencionó, a diferencia de décadas pasadas la tasa de crecimiento intercensal de los EF fue superior a la de la FF. Empero, si elimináramos Nuevo León de estos dos grupos, la tasa de crecimiento intercensal 1970-1980 del resto de entidades federativas sería de $2.8 \%$, casi la misma que la de la FF.

La razón de eliminar al estado de Nuevo León obedece a que su inclusión en el análisis demográfico fronterizo introduce un sesgo importante, pues si bien colinda con Estados Unidos, la población que habita en la FF (municipio de Anáhuac) es muy escasa. Esta representaba en 1980 $0.5 \%$ de la población total de la FF (véase cuadro 1), y $0.7 \%$ con respecto a la población de su estado. ${ }^{8}$

Nuevo León es sólo un ejemplo de lo impreciso que resulta abordar la problemática fronteriza a través de las entidades federativas, ya que sus indicadores socioeconómicos son también resultado de lo que sucede en otras partes de la entidad que no están tan inmersas en la interacción fronteriza; tal es el caso para este estado de la ciudad de Monterrey. Pero lo mismo podría decirse de otras entidades federativas: Hermosillo y Ciudad Obregón en Sonora; Torreón y Saltillo en Coahuila; Chihuahua en Chihuahua; y Tanipico y Ciudad Victoria en Tamaulipas, etcétera.

Otra dimensión que refuerza la aseveración anterior es el carácter urbano de la FF. En el cuadro 3 puede apreciarse la población que reside en localidades de más de 20000 habitantes en la República mexicana, en los EF y en la FF. En 1980, $49.8 \%$ y $68.1 \%$ de la población total del país y de los estados fronterizos respectivamente residía en localidades de más de 20000 habitantes, mientras que $77.9 \%$ lo hacía en la FF.

Más notable aún es que $83.8 \%$ de la población de la FF en 1980 residía en sólo diez de los treinta y ocho municipios (cuadro 1), y tres de ellos (Juárez, Mexicali y Tijuana) agrupaban aproximadamente 50\%. Así, la mayor concentración urbana en los municipios fronterizos en las entidades federativas del norte de México es otro ejemplo de las diferencias al abordar con una u otra unidad de análisis los fenómenos fronterizos.

El análisis de tasas de crecimiento y de concentración de población en localidades urbanas nos ha permitido ejemplificar de manera sencilla el distinto significado que tiene tomar a los municipios contiguos a la frontera versus las entidades federativas norteñas en el estudio demográfico fronterizo. La dinámica poblacional de la FF, además de mostrar caracte-

\footnotetext{
${ }^{7}$ Los datos de la Encuesta Demográfica de Baja California (1986) han arrojado evidencia sobre la significativa disminución que ha venido dándose, entre 1970 y 1986 , en lo que respecta a sus niveles de fecundidad, al observar el cambio tan drástico que ha habido en la estructura por edad y sexo de su población.

${ }^{8}$ En 1980 el estado de Nuevo León contaba con una población de 2513044 habitantes.
} 


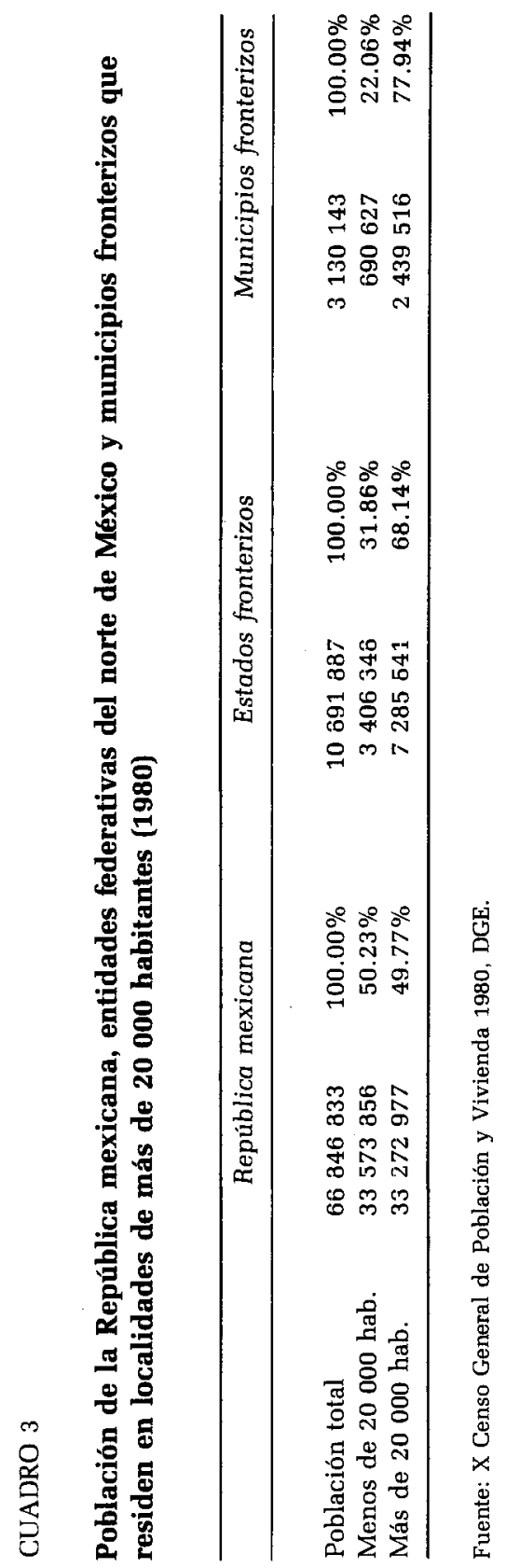


rísticas diferentes a la de los $\mathrm{EF}$, parece evidenciar más claramente las consecuencias de una relación fronteriza con el país económicamente más fuerte del mundo, y al que México ha estado ligado de forma dependiente durante muchos años.

La frontera norte de México, vista a través de los municipios fronterizos, ha presentado uno de los crecimientos demográficos del país más interesantes que han ocurrido durante el presente siglo, tanto por su elevada magnitud como por las causas que lo han condicionado. No obstante existe, por lo menos hasta 1980 , una evidente tendencia a la disminución en su ritmo de crecimiento, debido ante todo a los cambios ocurridos en los patrones migratorios hacia y desde la zona. Margulis y Tuirán (1984) han señalado que durante la década de los setenta, el crecimiento social de las ciudades fronterizas se redujo a niveles mínimos e incluso para algunas ciudades se registraron tasas negativas. Además, la emigración desde estas ciudades jugó un papel importante en la reducción de sus saldos netos migratorios. ${ }^{9}$

2. Niveles de marginación y características demográficas de los municipios fronterizos

Una vez expuestas las ventajas comparativas de tomar como contexto geográfico de la frontera norte de México a los municipios fronterizos, quisiéramos pasar a señalar algunos otros aspectos relevantes a su estudio. Uno de ellos se refiere a la diferencia que guardan éstos en cuanto a desarrollo socioeconómico, y el otro es abundar en el análisis de los fenómenos demográficos.

Las condiciones físico-geográficas y el tipo de desarrollo económico del norte de México han propiciado que más de tres cuartas partes de la población que reside en la FF se concentre en tan sólo diez de sus treinta y ocho municipios (distribuidos a lo largo de $2597 \mathrm{~km}$ de frontera), cuyas cabeceras municipales son importantes centros urbanos del país. ${ }^{10}$

Además de que en las principales ciudades se puede ver claramente la dimensión internacional de esta zona por su alto grado de interacción con Estados Unidos, esta situación demográfica ha traído como consecuencia que los indicadores socioeconómicos y demográficos de la población

${ }^{9}$ Muy difícilmente este patrón puede hacerse extensivo a lo que está ocurriendo actualmente en esta zona. Las condiciones económicas del pais han cambiado radicalmente durante los años ochenta. La crisis nacional parece volver a hacer emerger una fuerte inmigración a las principales ciudades fronterizas, las cuales han respondido de manera menos desafortunada ante la misma.

${ }^{10}$ Estos municipios son, de mayor a menor tamaño de población en 1980, Juárez, Mexicali, Tijuana, Matamoros, Reynosa, Nuevo Laredo, Ensenada, San Luis Río Colorado, Piedras Negras y Nogales. 
de la FF estén fuertemente determinados por sus características, propiciando a la vez que la mayoría de los estudios fronterizos se centren en el análisis de estos municipios y centros urbanos. Los resultados de estas investigaciones se hacen extensivos a toda la zona, sin diferenciar que son particulares del desarrollo de unos cuantos lugares.

Sin demeritar la importancia que tienen los centros urbanos de la frontera en la conformación de la $\mathrm{FF}$, creemos fundamental destacar la poca atención que se ha puesto en el estudio de sus municipios rurales. La razón implícita en su exclusión ha sido su escaso tamaño de población. Desde un punto de vista nacional estos municipios forman parte de la frontera norte de México, independientemente de su grado de interacción con Estados Unidos. Esto es más grave aún cuando - tal y como veremos más adelante- éstos son los municipios con mayor grado de marginación, y sobre los cuales las políticas y programas de desarrollo regional de México deberán prestar mayor atención durante los próximos años.

\subsection{Análisis de la marginación en la franja fronteriza norte de México}

Para ejemplificar la heterogeneidad interna en la FF tomamos como base uno de los pocos estudios que han utilizado información a nivel municipal en México; éste fue el realizado por la Coordinación General del Plan Nacional de Zonas Deprimidas y Grupos Marginados (Coplamar, 1982). El objetivo de dicho trabajo fue establecer una delimitación regional de la marginación en México que sirviera como marco de referencia para las estrategias de atención a los grupos marginados, basándose para ello en información de $1970 .{ }^{11}$

El estudio hecho por Coplamar permite derivar lo siguiente con respecto a la franja fronteriza: ${ }^{12}$

a) La FF es una de las zonas del país con menor grado de marginación o, visto de otra forma, una de las más desarrolladas en términos socioeconómicos.

b) De sus treinta y nueve municipios, únicamente cuatro fueron clasificados como núcleos marginados: Guerrero, Hidalgo y Jiménez en Coahuila y Manuel Benavides en Chihuahua ${ }^{13}$.

c) Dos municipios formaron parte del grupo de veinte municipios con menor marginación del país: Cananea y Nogales (ambos de Sonora).

${ }^{11}$ El concepto de marginación adoptado tuvo como finalidad caracterizar aquellos grupos que han quedado al margen de los beneficios del desarrollo nacional y de los beneficios de la riqueza generada, pero no necesariamente al margen de la generación de esa riqueza ni mucho menos de las condiciones que la hacen posible (Coplamar, 1982).

${ }^{12}$ Cabe señalar que el trabajo de Coplamar realizó el análisis para varias dimensiones: entidades federativas, regiones, municipios, zonas y núcleos marginados.

${ }^{13}$ Además de los 38 municipios que consideramos como fronterizos se agregó la congregación de Colombia del estado de Nuevo León, ya que era fronterizo en 1970. 
Los resultados de Coplamar corroboran la ubicación de la FF como una de las zonas con mejores condiciones de vida del país. Sin embargo, debido a que dicho trabajo ịcrarquizó la marginación en función de las medias nacionales de los indicadores socioeconómicos, y a que nuestro interés se centraba en establecer las diferencias internas en el contexto fronterizo, fue que decidimos reconstruir la metodología de Coplamar trabajando únicamente con los municipios fronterizos, de tal manera que el grado de marginación se estableciera con respecto a los valores promedio de la FF y no del país. Esto fue posible gracias a que los indicadores a nivel municipal fueron publicados por Coplamar.

Debemos mencionar que la desventaja más importante de tomar como base este trabajo es la limitación temporal de los datos, ya que éstos caracterizan la situación socioeconómica existente 18 años atrás. Desafortunadamente no tuvimos tiempo suficiente para actualizar la información necesaria. Además, de que esta tarea hubiera tenido los inconvenientes de trabajar con el censo de 1980, el cual presentó serias deficiencias en su información.

Así, para medir las características de la marginación en la FF utilizamos, al igual que Coplamar, los siguientes 19 indicadores:

-Generales: 1. porcentaje de población económicamente activa (PEA) que percibe ingresos inferiores a 1000 pesos mensuales, 2. porcentaje de PEA subempleada, 3. porcentaje de población rural, 4. porcentaje de PEA en el sector agropecuario, 5. porcentaje de población rural incomunicada.

-Alimentación: 6. porcentaje de población que consume leche dos o menos días a la semana, 7. porcentaje de población que consume carne dos o menos días a la semana, 8. porcentaje de población que consume huevo dos o menos días a la semana.

-Educación: 9. porcentaje de población analfabeta de 10 y más años de edad, 10. porcentaje de población de 12 y más años sin primaria completa.

- Salud: 11. tasa de mortalidad general, 12. tasa de mortalidad preescolar, 13. habitantes por médico.

-Vivienda y sus servicios: 14. porcentaje de viviendas sin agua entubada, 15. Porcentaje de viviendas de uno y dos cuartos, 16. porcentaje de viviendas $\sin$ electricidad, 17. porcentaje de viviendas sin drenaje.

-Otras necesidades: 18. porcentaje de población de un año y más que no usa calzado, 19. porcentaje de viviendas sin radio ni televisión.

Para calcular los índices de marginación se utilizó la siguiente fórmula:

$$
\mathrm{IM}_{\mathrm{j}}=\sum_{\mathrm{i}=1}^{19} \mathrm{FP}_{\mathrm{i}} \mathrm{X}_{\mathrm{ij}}-\overline{\mathrm{X}}_{\mathrm{i}} \quad \mathrm{j}=1 \ldots \mathrm{N}
$$


donde:

$\mathrm{IM}_{\mathrm{j}}=$ índice de marginación de la unidad geográfica $\mathrm{j}$

$\mathrm{X}_{\mathrm{ij}}=$ valor de indicador $\mathrm{i}$, de la unidad geográfica $\mathrm{j}$

$X_{i}=$ valor de la media del indicador $i$

$\mathrm{d}_{\mathrm{i}}=$ desviación estándar del indicador $\mathbf{i}$

$F P_{i}=$ factor de ponderación del indicador $\mathrm{i}$

$\mathrm{N}=39$ en el caso de la FF.

Debido al tipo de indicadores utilizados, el valor del índice se mueve en sentido directo al grado de marginación; esto es, a mayor valor del índice mayor marginación, y viceversa. En la estimación del factor de ponderación de cada indicador $\left(\mathrm{FP}_{\mathrm{i}}\right)$ se utilizó el método de componentes principales (análisis factorial).

En el cuadro 4 pueden observarse las ponderaciones de cada uno de los indicadores de marginación para la FF, así como las obtenidas por Coplamar a nivel municipal para todo el país. ${ }^{24}$ Los indicadores de mayor peso en la explicación del índice de marginación para la FF fueron: población adulta sin primaria (0.96), viviendas sin electricidad (0.93), PEA agrícola (0.90), población rural (0.89), viviendas sin drenaje $(0.88)$ y subconsumo de carne (0.85). Los indicadores de menor peso fueron incomunicación rural (0.22) y población sin calzado (0.22). Las diferencias obtenidas con respecto a los resultados presentados a nivel nacional son considerables (véase el cuadro 4).

Una vez estimados los ponderadores, así como la media y desviación estándar de cada uno de los indicadores para la franja fronteriza, se calcularon los índices de marginación. ${ }^{15}$ Éstos se presentan ordenados y estratificados, de menor a mayor marginación, en el cuadro 5.

Los índices de marginación mostraron una variación desde -9.40 en el municipio de Cananea (menor marginación), hasta 20.81 en Manuel Benavides (mayor marginación). Como la diferencia era de casi treinta puntos decidimos dividir los índices en tres estratos. El estrato de margina-

\footnotetext{
${ }^{14}$ El vector de ponderación asociado a la primera componente explicó el $48.8 \%$ de la varianza total en la franja fronteriza.

${ }^{15}$ Cabe señalar que en la reproducción de la metodología utilizada por Coplamar para la $\mathrm{FF}$, la principal dificultad presentada fue la estimación de la media $\left(\mathrm{X}_{\mathrm{i}}\right)$ y de la desviación estándar $\left(\mathrm{d}_{\mathrm{i}}\right)$ de los indicadores. Conocíamos para cada municipio los valores de los indicadores $\left(\mathrm{X}_{\mathrm{ij}}\right)$ y los denominadores para su construcción. Por ejemplo, se tenía el indicador, porcentaje de PEA de bajos ingresos, y el denominador del mismo (PEA total). Dado que no se podia estimar la media a partir de los mismos indicadores, lo que se hizo fue calcular el número absoluto de la variable PEA con bajos ingresos para cada municipio (multiplicando la PEA total por el porcentaje de PEA de bajos ingresos). Con esto se estimó el valor de la variable para toda la franja fronteriza, lo cual junto con la PEA total de la zona, permitió calcular la media del indicador mencionado. Esto se realizó para cada uno de los 19 indicadores. Una vez conocidos el valor de $\mathrm{X}_{\mathrm{i}} \mathrm{y}$ de cada $\mathrm{X}_{\mathrm{ij}}$ se pudo obtener la desviación estándar de cada indicador.
} 
CUADRO 4

Ponderación de los indicadores de marginación para la franja fronteriza y la República mexicana

\begin{tabular}{lcc}
\hline & $\begin{array}{c}\text { Nivel municipal } \\
\text { franja }\end{array}$ & $\begin{array}{c}\text { Nivel municipal } \\
\text { República Me- } \\
\text { xicana }\end{array}$ \\
\hline Bajos ingresos & 0.71 & 0.29 \\
Subempleo & 0.32 & 0.07 \\
Población rural & 0.89 & 0.61 \\
PEA agrícola & 0.90 & 0.79 \\
Incomunicación rural & 0.22 & 0.29 \\
Subconsumo de leche & 0.79 & 0.87 \\
Subconsumo de carne & 0.85 & 0.79 \\
Subconsumo de huevo & 0.75 & 0.67 \\
Analfabetismo & 0.67 & 0.63 \\
Población sin primaria & 0.96 & 0.88 \\
Mortalidad general & -0.37 & 0.39 \\
Mortalidad preescolar & -0.23 & 0.54 \\
Habitantes por médico & 0.60 & 0.37 \\
Viviendas sin agua & 0.73 & 0.70 \\
Viviendas con 1 y 2 cuartos & 0.75 & 0.71 \\
Viviendas sin electricidad & 0.93 & 0.80 \\
Viviendas sin drenaje & 0.88 & 0.72 \\
Población sin calzado & 0.22 & 0.60 \\
Viviendas sin radio y t.v. & 0.65 & 0.83 \\
Porcentaje de varianza asociado & & \\
$\quad$ a la primera componente & 48.8 & 41.8 \\
\hline
\end{tabular}

Fuênte: cálculos hechos por los autores y Coplamar (1982).

ción baja se conformó por los municipios cuyo valor fue de signo negativo; los de marginación media quedaron constituidos por los que tuvieron valores de 0.01 a 9.99, y los de alta marginación por los de 10.00 y más. ${ }^{16}$

Las diferencias en los grados de marginación resultan bastante elocuentes. Únicamente once de los municipios comprendían el estrato de marginación baja en 1970. Llaman la atención Cananea, Mier, Naco, Agua Prieta y Miguel Alemán debido a que no son reconocidos normalmente entre los municipios fronterizos más importantes. A reserva de conocer

${ }^{16}$ Hubiese sido conveniente estratificar con los mismos criterios que Coplamar. Sin embargo, las diferencias presentadas entre los valores más altos y bajos de los índices de marginación entre aquel trabajo y éste, no permitieron que esto se realizara. La estratificación de Coplamar fue la siguiente: marginación baja de -20 y menos; media baja de -10 a -19.99 ; media de -0.01 a -9.99 ; media alta de 0.01 a 9.99 y alta de 10 y más (Coplamar, 1982). 
CUADRO 5

Índices de marginación de los municipios fronterizos agrupados por estratos

\begin{tabular}{|c|c|c|c|}
\hline Municipio & $\begin{array}{l}\text { Índice de } \\
\text { margi- } \\
\text { nación }\end{array}$ & Estratos & $\begin{array}{c}\text { Grado de } \\
\text { margi- } \\
\text { nación }\end{array}$ \\
\hline Cananea, Son. & -9.40 & & \\
\hline Nogales, Son. & -7.79 & & \\
\hline Piedras Negras, Coah. & -4.19 & & \\
\hline Tijuana, B.C. & -4.15 & & \\
\hline Mier, Tamps. & -3.59 & & \\
\hline Nuevo Laredo, Tamps. & -3.29 & -0.01 y menos & Bajo \\
\hline Naco, Son. & -3.06 & & \\
\hline Juárez, Chih. & -2.65 & & \\
\hline Agua Prieta, Son. & -1.24 & & \\
\hline Reynosa, Tamps. & -0.91 & & \\
\hline Miguel Alemán, Tamps. & -0.48 & & \\
\hline Ensenada, B.C. & 0.26 & & \\
\hline Mexicali, B.C. & 0.43 & & \\
\hline Acuña, Coah. & 0.64 & & \\
\hline San Luis Río Colorado, Son. & 0.73 & & \\
\hline Tecate, B.C. & 1.67 & & \\
\hline Matamoros, Tamps. & 1.91 & & \\
\hline Guerrero, Tamps. & 3.15 & & \\
\hline Puerto Peñasco, Son. & 4.08 & 0.01 a 9.99 & Medio \\
\hline Altar, Son. & 4.51 & & \\
\hline Santa Cruz, Son. & 4.74 & & \\
\hline Caborca, Son. & 5.63 & & \\
\hline Anáhuac, N.L. & 6.42 & & \\
\hline Valle Hermoso, Tamps. & 8.71 & & \\
\hline Gustavo Díaz Ordaz, Tamps. & 8.83 & & \\
\hline Río Bravo, Tamps. & 9.72 & & \\
\hline Camargo, Tamps. & 10.03 & & \\
\hline Ojinaga, Chih. & 10.30 & & \\
\hline Ascensión, Chih. & 10.87 & & \\
\hline Saric, Son. & 11.23 & & \\
\hline Guadalupe, Chih. & 12.03 & & \\
\hline Colombia, N.L. & 13.82 & 10.00 y más & Alto \\
\hline Práxedis G. Guerrero, Chih. & 14.17 & & \\
\hline Janos, Chih. & 16.84 & & \\
\hline Guerrero, Coah. & 17.72 & & \\
\hline Hidalgo, Coah. & 17.80 & & \\
\hline Jiménez, Coah. & 17.90 & & \\
\hline Ocampo, Coah. & 17.96 & & \\
\hline Manuel Benavides, Chih. & 20.81 & & \\
\hline
\end{tabular}

Fuente: cálculos hechos por los autores. 
CUADRO 6

Índices de marginación y grado de marginación de los municipios fronterizos agrupados por entidades federativas

\begin{tabular}{|c|c|c|c|}
\hline & Municipio & $\begin{array}{c}\text { Índice de } \\
\text { marginación }(F F)\end{array}$ & $\begin{array}{l}\text { Grado de } \\
\text { marginación }\end{array}$ \\
\hline \multirow{4}{*}{ Baja California } & a Tijuana & -4.15 & Bajo \\
\hline & Ensenada & 0.26 & Medio \\
\hline & Mexicali & 0.43 & Medio \\
\hline & Tecate & 1.67 & Medio \\
\hline \multirow[t]{6}{*}{ Coahuila } & Piedras Negras & -4.19 & Bajo \\
\hline & Acuña & 0.64 & Medio \\
\hline & Guerrero & 17.72 & Alto \\
\hline & Hidalgo & 17.80 & Alto \\
\hline & Jiménez & 17.90 & Alto \\
\hline & Ocampo & 17.96 & Alto \\
\hline \multirow[t]{7}{*}{ Chihuahua } & Juárez & -2.65 & Bajo \\
\hline & Ojinaga & 10.30 & Alto \\
\hline & Ascensión & 10.87 & Alto \\
\hline & Guadalupe & 12.03 & Alto \\
\hline & Práxedis G. Guerrero & 14.17 & Alto \\
\hline & Janos & 16.84 & Alto \\
\hline & Manuel Benavides & 20.81 & Alto \\
\hline \multirow[t]{2}{*}{ Nuevo León } & Anáhuac & 6.42 & Medio \\
\hline & Colombia & 13.82 & Alto \\
\hline \multirow[t]{10}{*}{ Sonora } & Cananea & -9.40 & Bajo \\
\hline & Nogales & -7.79 & Bajo \\
\hline & Naco & -3.06 & Bajo \\
\hline & Agua Prieta & -1.24 & Bajo \\
\hline & San Luis Río Colorado & 0.73 & Medio \\
\hline & Puerto Peñasco & 4.08 & Medio \\
\hline & Altar & 4.51 & Medio \\
\hline & Santa Cruz & 4.74 & Medio \\
\hline & Caborca & 5.63 & Medio \\
\hline & Saric & 11.23 & Alto \\
\hline \multirow[t]{10}{*}{ Tamaulipas } & Mier & -3.59 & Bajo \\
\hline & Nuevo Laredo & -3.29 & Bajo \\
\hline & Reynosa & -0.91 & Bajo \\
\hline & Miguel Alemán & -0.48 & Bajo \\
\hline & Matamoros & 1.91 & Medio \\
\hline & Guerrero & 3.15 & Medio \\
\hline & Valle Hermoso & 8.71 & Medio \\
\hline & Gustavo Díaz Ordaz & 8.83 & Medio \\
\hline & Río Bravo & 9.72 & Medio \\
\hline & Camargo & 10.03 & Alto \\
\hline
\end{tabular}


más sobre las condiciones económicas y sociales de estos municipios, no debe descartarse la posibilidad de tener problemas con la calidad de la información a un nivel tan desagregado como es el municipal.

Es de resaltar que los municipios de las principales ciudades fronterizas (Tijuana, Juárez, Nogales, Mexicali, Ensenada, Reynosa, Nuevo Laredo, Piedras Negras y Matamoros) muestran cierta congruencia en la estimación de sus índices, ya que se encuentran en los grupos de baja y media marginación.

A excepción de Juárez en Chihuahua y Piedras Negras y Acuña en Coahuila, los municipios de estos estados presentaron un alto grado de marginación (ver cuadro 6). Inclusive, diez de los trece municipios del estrato de mayor marginación se concentran en las entidades federativas mencionadas (ver cuadro 5). Los municipios de Baja California, Sonora y Tamaulipas muestran en casi su totalidad grados de marginación bajos y medios.

En resumen podemos decir lo siguiente:

i) Los resultados de los índices de marginación confirman la existencia de una gran heterogeneidad al interior en la franja fronteriza en cuanto a desarrollo socioeconómico. A estas diferencias habría que añadir la presencia de un fuerte desbalance en la distribución geográfica de la marginación, ya que los municipios con mayor marginación pertenecen en su mayoría a los estados de Chihuahua y Coahuila.

ii) Los distintos grados de marginación que presentan los municipios fronterizos, aunados a los problemas de integración, muestran la complejidad de considerar a esta zona como una región homogénea.

iii) Aunque los resultados revelan las diferencias que imperaban en 1970, creemos muy difícil que la brecha existente entre los municipios más marginados y los menos marginados se haya cerrado significativamente en los últimos años.

\subsection{Algunas características demográficas generales de los municipios fronterizos}

Con el fin de complementar el panorama de las diferencias que existen al interior de la FF, en el cuadro 7 se presentan algunos indicadores demográficos generales para 1980. Esta vez tratando de relacionarlos con su grado de marginación.

La mitad de los municipios con alto grado de marginación en 1970 experimentaron bajas tasas de crecimiento demográfico en la década 19701980; inclusive para seis de ellos éstas fueron negativas. Aunque no existe uniformidad en la magnitud de las tasas al interior de los estratos, todos los municipios con baja marginación mostraron crecimientos positivos. En el estrato de marginación media la mayoría presentó tasas de crecimiento altas. Únicamente en tres de ellos se redujo su población total. 
Por otra parte, aunque la tendencia no es del todo clara, se puede ver cómo los municipios con mayor marginación presentaron altos índices de masculinidad con respecto a los de menor grado de marginación. La diferencia es abismal entre Juárez (92.87) e Hidalgo (128.96). Mientras que ningún municipio con bajo grado de marginación presentó un índice mayor o igual a cien en 1980, casi la totalidad de los de marginación alta rebasan esa cifra.

Cabe destacar, por ser completamente atípico, el caso de Santa Cruz cuyo índice de masculinidad es de 122.55, y está considerado dentro del grupo de marginación media. No obstante, la tasa de crecimiento de este municipio fue negativa durante la década 1970-1980, una característica común, junto con altos índices de masculinidad, de los municipios que conforman el estrato de marginación alta.

Con todas las limitaciones que implica trabajar con estos indicadores demográficos tan burdos, ${ }^{17} \mathrm{y}$ sin desconocer las deficiencias que la información puede tener a un nivel tan desagregado como el municipal, podríamos decir que las tasas de crecimiento negativas (o muy cercanas a cero) parecen evidenciar una expulsión importante de población de los municipios con mayor marginación. Esta emigración muy probablemente se dirigió hacia los municipios fronterizos más cercanos y con menos problemas socioeconómicos.

Si relacionamos la hipótesis anterior con la información de los índices de masculinidad, podemos suponer que los valores altos de este indicador en los municipios más marginados, proporcionan una imagen en que la mujer es la que interviene básicamente en la emigración. Este comportamiento puede asociarse con la fuerte demanda de fuerza de trabajo femenina que existe en las ciudades fronterizas más desarrolladas (véase Cruz y Zenteno, 1987). A la vez, la estructura económica rural de los lugares con mayor marginación podría estar provocando una necesidad de retener más a la población masculina, con el fin de continuar desarrollando las labores del campo.

Por último, la razón niños-mujer (medida indirecta de fecundidad) no parece mostrar una relación muy definida con los estratos de marginación. Sólo para dos de ellos se puede hablar de una posible asociación. La razón niños-mujer manifiesta la presencia de una menor fecundidad en los municipios con baja marginación y más alta en aquéllos cuyo grado de marginación es alto. Sin embargo, la migración femenina puede estar sesgando fuertemente cualquier conclusión que se desee derivar a través de la razón niños-mujer, especialmente en los casos en que existe más marginación.

17 Muy sensibles por ejemplo al tamaño tan reducido de las cifras de algunos municipios fronterizos. 


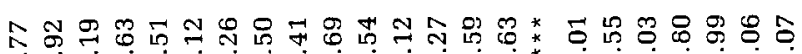

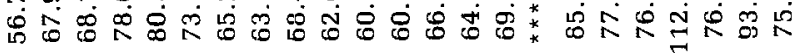

क्ष

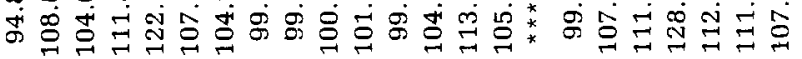

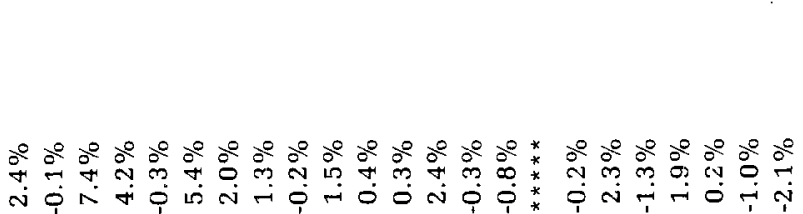

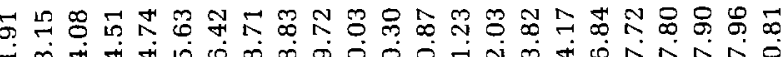

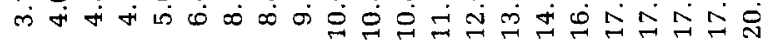

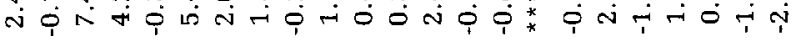




\section{Consideraciones finales}

La oportunidad de desarrollar una investigación demográfica regional para la frontera norte de México implica la resolución de varios problemas teórico-metodológicos. No se trata simplemente de reproducir los estudios demográficos nacionales para un agregado de estados o municipios. Esto significa trasladarse hasta la naturaleza de los fenómenos fronterizos y del carácter regional de los mismos. Mucho queda por hacer al respecto. Por lo pronto, este trabajo intentó avanzar en algunas ideas que creemos relevantes.

Debido a que el concepto de "región fronteriza" ha sido acuñado más por el reconocimiento de problemáticas específicas en el norte del país (inherentes en su mayoría a convivir intensamente en un espacio fronterizo con Estados Unidos), y a que estos hechos tienen lugar en áreas cuya integración muchas veces es nula, es que mientras no se estudie con mayor detenimiento las regiones fronterizas, cualquier delimitación que defina globalmente un espacio para el estudio de los fenómenos fronterizos debe ser considerado un contexto geográfico más que una región. En este sentido, y para el caso de la investigación demográfica, hemos mostrado que el conjunto de municipios pegados a la línea divisoria con Estados Unidos resultan ser un contexto fronterizo más adecuado.

Al trabajar con este marco geográfico debemos tomar muy en cuenta los riesgos que se corren al generalizar sobre sus características sociales, económicas, demográficas, etc., ya que los municipios fronterizos guardan entre sí diferencias importantes, tal y como pudimos ejemplificar a través del análisis de algunos indicadores demográficos.

\section{Bibliografía}

Bassols, Ángel. Geografía económica de México, Trillas, 3a. ed., México, 1978. Bataillon, Claude. La regiones geográficas de México, Siglo XXI Editores, México, 1969.

Coordinación General del Plan Nacional de Zonas Deprimidas y Grupos Marginados (Coplamar). Necesidades esenciales en México: Situación actual y perspectivas al año 2000, Geografía de la marginación, Coplamar y Siglo XXI, México, 1982.

Corona, Rodolfo. Evaluación de los datos censales de 1980, Población residente y migración en Baja California, Centro de Estudios Fronterizos del Norte de Mexico, Tijuana, B.C., Mexico, 1986.

Cruz, Rodolfo y René Zenteno, "Algunas características de la población económicamente activa femenina en Tijuana", presentado en el Coloquio sobre Fuerza de Trabajo Femenina en México, México, D.F., 1987 (en prensa).

Driscoll, Bárbara. El Programa de Braceros Ferroviarios,Centro de Estudios Fronterizos̀ del Norte de México, Tijuana, B.C., México, 1985. 
Fernández, Raúl. "Las reformas a la inmigración y su impacto en la frontera MéxicoEstados Unidos, en Estudios Fronterizos, vol. 1, núm. 4-5, 1984.

García, Víctor. "La economía mexicana y la economía fronteriza del norte de México", en La Frontera del Norte, Roque González Salazar (compilador), El Colegio de México, México, 1981.

Gobierno Constitucional de los Estados Unidos Méxicanos, Programa de desarrollo de la frontera norte, México, 1985.

Margulis, Mario y Rodolfo Tuirán, Desarrollo y población en la frontera norte: el caso de Reynosa, El Colegio de México, México, 1986.

"Nuevos patrones migratorios en la frontera norte: la emigración", en Demografía y Economía, vol. XVIII, núm. 59, 1984.

Mendoza, Eliseo. "Historia de los programas federales para el desarrollo económico de la frontera norte, en Administración del desarrollo de la frontera norte, El Colegio de México, México, 1982.

Ojeda, Mario. 'Presentación", en Administración del desarrollo de la frontera norte, El Colegio de México, México, 1982.

Revel-Mouroz, Jean. "La frontera México-Estados Unidos: Mexicanización e internacionalización", en Estudios Fronterizos, vol. 1, núm 4-5, 1984.

Tamayo, Jesús y José Luis Fernández. Zonas fronterizas (México-Estados Unidos), Centro de Investigación y Docencia Económica (CIDE), México, 1983.

Uniquel, Luis, et al. El desarrollo urbano de México: Diagnóstico e implicaciones futuras, El Colegio de México, México, 1976.

Urquidi, Víctor y Sofía Méndez. "Importancia de la zona fronteriza del norte de México", en Foro Internacional, vol. XVI-2, octubre-diciembre, 1975.

Victoria, Edmundo. "Características del desarrollo económico de la franja fronteriza norte de México", en Natural Resources Journal, vol. 22, núm. 4, octubre de 1982. 
